\title{
ANÁLISIS
}

\section{Tendencias recientes de la integración asiática y la participación de Japón ${ }^{1}$}

DOI: 10.32870/mycp.v14i42.371

Mitsuhiro Kagami

\section{Resumen}

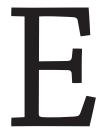

1 este asiático (ASEAN 10 + Australia, China, India, Japón, Corea y Nueva Zelanda, en este trabajo) se ha convertido en un importante centro de producción mundial, así como en uno de los más grandes mercados de consumo del mundo. En 2008, esta región contribuyó con $33 \%$ de las exportaciones mundiales y $19 \%$ de las importaciones, respectivamente. El mercado de consumo de esta región está creciendo debido a que la población es muy grande (3,245 millones en el año 2008) además del rápido crecimiento de la clase media. Esta tendencia será aún más acelerada debido a la formación de la Comunidad Económica de la ASEAN en 2015 y los TLC bilaterales recientemente establecidos entre ASEAN y sus seis países vecinos. De esta manera, ASEAN se convierte en un pivote o eje de comercio, servicios e inversiones en el este asiático. Vale la pena mencionar, que la inclusión de Rusia y los Estados Unidos como miembros de la Cumbre del Este Asiático a partir de 2011, va a tener impactos enormes, no sólo en las relaciones geopolíticas de poder en los países relacionados, sino también en el Acuerdo de la Asociación Transpacífica y el Área de Libre Comercio de Asia-Pacífico de la APEC.

Palabras clave: integración económica, Asia, TLC, la Comunidad Económica de la ASEAN, Japón

1. Artículo recibido el 11 de marzo de 2011 y dictaminado el 06 de mayo de 2011.

2. Profesor de Teiko University. Department of Economics. 


\section{Abstract}

East Asia (ASEAN 10 plus Australia, China, India, Japan, Korea and New Zealand in this paper) has become a world production center as well as one of the largest consumer markets in the world. Exports of final goods as a share of world total from this region reached to 33 percent and that of imports to 19 percent in 2008. The consumer market in this region is expanding thanks to its huge population (3,245 million in 2008) with fast growing middle class. The tendency will further be accelerated due to the formation of the ASEAN Economic Community (AEC) in 2015 and recently established bilateral FTA between ASEAN and its six neighboring countries. Thus, ASEAN becomes a pivot or hub of trade, services and investment in East Asia. It is worth mentioning that the inclusion of Russia and the United States to the East Asian Summit members from 2011 will have an enormous impact to geopolitical and power relations among related nations as well as the Trans-Pacific Partnership (TPP) and the Free Trade Area of Asia-Pacific (FTAAP) of APEC.

Keywords: economic integration, Asia, FTA, ASEAN Economic Community, Japan

\section{Introducción}

El mundo cambia en términos de producción y consumo. En el pasado, los países avanzados fabricaban productos finales y los exportaban a los países en vías de desarrollo. Hoy, somos testigos de una transformación. Las economías en desarrollo de Asia, como China, los Estados que integran la Asociación de Naciones del Sudeste de Asia (ASEAN) ${ }^{3}$ y la India se han convertido en fábricas para el mundo y, al mismo tiempo, en un enorme mercado consumidor. Este cambio se ve acelerado por el llamado shock de Lehman, la crisis financiera europea en relación con Grecia, el titubeo de Japón provocado por la incertidumbre política y los efectos del terremoto y el tsunami ocurrido en marzo de 2011, y la crisis nuclear en la central de Fukushima. Mientras tanto, los países asiáticos, incluyendo las economías de Oceanía, tratan de lograr un Tratado de Libre Comercio (TLC) y realizan esfuerzos de integración, lo que ha traído como resultado una rápida liberalización del comercio entre ellos.

3. Estados que integran ASEAN: Brunei Darussalam, Camboya, Filipinas, Indonesia, Laos, Malasia, Myanmar, Singapur, Tailandia y Vietnam. 
La primera sección de este trabajo relata la cambiante oferta y demanda mundial de productos finales e intermedios. La segunda explica el proceso de liberalización del comercio y los esfuerzos de integración en Asia. La tercera aborda la participación de Japón en tales movimientos. Posteriormente se presentan algunas conclusiones.

\section{Fábrica del mundo y grandes mercados consumidores}

Si clasificamos los productos comerciales en primarios, intermedios y finales, descubriremos un resultado asombroso en términos de cambio de la base productiva y de los mercados consumidores en el mundo, utilizando los datos de las Naciones Unidas sobre el Comercio de Productos de Consumo (UN Comtrade). Para fabricar productos finales se necesitan productos intermedios. La participación mundial de la ASEAN+6 (ASEAN, China incluyendo Hong Kong, Japón, Corea, India, Australia y Nueva Zelanda) con respecto a las importaciones de productos intermedios aumentó de $27 \%$ en 2000 a 30\% en 2008 , mientras la de las exportaciones de productos finales también aumentó de $30 \%$ a $33 \%$ durante el mismo periodo. Ello significa que esta región exporta al mundo aproximadamente un tercio de los productos finales, por lo que constituye una fábrica mundial.

En la actualidad, esta región (el este asiático, en este trabajo) posee aproximadamente $49 \%$ de la población mundial y alrededor de $25 \%$ del PBI mundial. En especial, el cambio ocurrido en China, incluyendo Hong Kong, es asombroso. Su participación en la importación mundial de productos intermedios cambió de $8.7 \%$ en 2000 a $11.6 \%$ en 2008, mientras su participación en la exportación de productos finales aumentó significativamente de $10.7 \%$ a 17.3\% durante el mismo periodo.

Otro hecho que podría resultar interesante es el cambio que tiene lugar en la demanda mundial y los mercados consumidores. Desde el punto de vista de la demanda de productos finales, se reconoce que se está produciendo un cambio de los mercados importadores. La participación de la región en términos de importaciones de productos finales aumentó de $18.9 \%$ en 2000 a 19.2\% en 2008. Por el contrario, la participación de EU disminuyó drásticamente de $24 \%$ a $16 \%$ durante el mismo lapso. Esto demuestra que el este asiático está emergiendo como uno de los mayores mercados consumidores del mundo. Este cambio crucial ocurre tanto en el comercio global como en los mercados mundiales, y continuará ocurriendo, si tenemos en cuenta la 
enorme población de China y la India y el surgimiento de la clase media en estos países asiáticos.

\section{Liberalización del comercio e inversión e integración en el este asiático}

ASEAN

La ASEAN, o Asociación de Naciones del Sudeste de Asia, se creó en 1967 con cinco países (Indonesia, Malasia, Filipinas, Singapur y Tailandia); en un inicio, para luchar contra la infiltración del comunismo. El grupo pasó de ser una asociación política a una más económica y en 1984 se incorporó Brunei Darussalam. Posteriormente, en el decenio de 1990, los países de la cuenca del río Mekong se hicieron miembros de la ASEAN: Vietnam en 1995, Myanmar y RDP Lao en 1997, y Camboya en 1999. En 1992, los miembros de la ASEAN decidieron formar un área de libre comercio en 15 años sobre la base de la Declaración de Singapur, y decidieron llamarla Área de Libre Comercio de la ASEAN (AFTA, por sus siglas en inglés). Este acuerdo entró en vigor en 1993, en principio con la intención de reducir progresivamente los aranceles en la región hasta 2008.

El AFTA no es una unión aduanera con un arancel común sobre las importaciones. Para los productos fabricados dentro de la región de la ASEAN, los miembros deben aplicar un arancel de 0\% a 5\% (a los últimos en integrarse a la ASEAN: Vietnam, Myanmar, RDP Lao y Camboya se les concedió un periodo adicional para poner en práctica las reducciones de los aranceles). Esto se conoce como Acuerdo de Arancel Preferencial Común Efectivo (CEPT, por sus siglas en inglés). De manera general, los productos fabricados en la región deberán incluir al menos $40 \%$ de contenido local de la ASEAN.

También se iniciaron otras actividades conocidas como "AFTA Plus", tales como la eliminación de las barreras no arancelarias, la armonización de códigos arancelarios y el desarrollo de normas comunes de certificación de los productos. Además, se liberalizará el comercio de servicios (Acuerdo Marco para los Servicios de la ASEAN, 1995). En 2003 se aceleró el proceso de reducción de aranceles y los miembros de la ASEAN acordaron aplicar cero aranceles prácticamente a todas las importaciones en 2010 para los miembros fundadores de la asociación y en 2015 para los cuatro miembros incorporados 
posteriormente. ${ }^{4}$ Desde entonces, el CEPT ha aumentado enormemente el comercio intrarregional de la ASEAN.

Los objetivos de integración de la ASEAN se presentaron por primera vez en 1997 como "Visión 2020 de la ASEAN". La meta era integrar la Comunidad Económica de la ASEAN (AEC, por sus siglas en inglés), la Comunidad de Seguridad de la ASEAN (ASC, por sus siglas en inglés), y la Comunidad Sociocultural de la ASEAN (ASCC, por sus siglas en inglés). Esto se ratificó en la Cumbre de la ASEAN celebrada en Bali en 2003 (Bali Concord II). Especialmente, para 2015 se debe crear una AEC, y la Declaración de Cebu, firmada en 2007, reafirma un compromiso de acelerar el establecimiento de una comunidad integrada de la ASEAN teniendo en cuenta el progreso favorable del AFTA. En la Cumbre de la ASEAN celebrada en Singapur en noviembre de 2007 se presentó el "Anteproyecto de la Comunidad Económica de la ASEAN" que mostraba el deseo de crear una AEC.

El "Anteproyecto" (tabla 1) contiene cuatro pilares: 1. Mercado único y base de producción, 2. Región económica competitiva, 3. Desarrollo económico equitativo, y 4. Integración a la economía global. El primer pilar implica liberalizar la región en términos del comercio de productos y servicios (o el logro pleno de la CEPT-AFTA), y por consiguiente, transformar la región en la fábrica de producción del mundo así como en un mercado único. El segundo pilar establece una atmósfera más competitiva promoviendo una política de competencia, protección al consumidor, derechos sobre la propiedad intelectual, desarrollo de infraestructura, reforma fiscal y comercio electrónico. El tercer pilar está dirigido a reducir la diferencia de desarrollo entre los últimos países que se incorporaron a la ASEAN y los primeros en hacerlo. Programas de puesta al día de los llamados países CLMV o Camboya, RDP Lao, Myanmar y Vietnam se diseñan de acuerdo con el desarrollo de pequeñas y medianas empresas y la Iniciativa para la Integración de la ASEAN. El cuarto pilar aspira a que la ASEAN se integre sin problemas a la economía

4. La AFtA-CEPT se convirtió en ATiga (Acuerdo de Comercio de Productos de la ASEAN), firmado en febrero de 2009. El ATIGA exige ahora que los seis miembros originales de la ASEAN cumplan con el $0 \%$ de aranceles para los productos de la Lista de Inclusión (IL, por sus siglas en inglés) y de 0\%-5\% para los productos de las Lista Sensible (SL, por sus siglas en inglés) y de la Lista Muy Sensible (HSL, por sus siglas en inglés) en el año 2010. Para los países de CLMV, es $0 \%$ para los productos IL en el año 2015 , y $0 \%-5 \%$ para los productos de la SL y la HSL en el año 2017 y algunos productos de exclusión en el año 2018, dependiendo del país. 
mundial, por ejemplo a través de los efectos de sinergia de los TLC, y a que la región participe en la cadena de suministro global.

El "Anteproyecto" presenta un cronograma estratégico para cada paso de la política para el avance de la AEC de 2008 a 2015 de la forma en que lo planificó la Secretaría de la ASEAN. ${ }^{5}$ También en 2007, la Cumbre de la ASEAN en Singapur aprobó unos "Estatutos de la ASEAN" para regular los procedimientos de la secretaría, la presidencia, los embajadores residentes, la solución de disputas, la creación de una organización de derechos humanos, y otras reglas importantes para los países miembros.

\section{La ASEAN como un centro de comercio e inversión}

Considerando el potencial de crecimiento rápido, el formidable progreso de la liberalización, y la gran población de aproximadamente 600 millones en los 10 países que integran la ASEAN, las naciones vecinas no podían ignorar la capacidad económica de la región como socio comercial. El primer paso lo dio China, luego Japón, Corea del Sur (en adelante Corea), Australia y Nueva Zelanda, e India. Ello significa que la ASEAN se convirtió en un centro comercial de Asia. La verdadera razón detrás de esto es el tratamiento discriminatorio de los aranceles. Por ejemplo, Corea tiene un TLC con la ASEAN, pero Japón no.

A través de ese TLC, una fábrica coreana en Tailandia puede importar paneles LCD de Corea sin pagar aranceles y exportar los TV terminados a otros países de la ASEAN también sin pagar aranceles utilizando el AFTA. Por otra parte, una fábrica japonesa en Tailandia tiene que pagar aranceles por su importación de paneles LCD de Japón. Por consiguiente, Japón pierde la competitividad de precios en el mercado de la ASEAN contra Corea. Por tanto, los países vecinos firmaron TLC con la ASEAN para no perder la competitividad de precios. Esto creó un auge de TLC con la ASEAN por parte de los países vecinos en lo que se ha dado en llamar "efecto de subirse al tren". ${ }^{6}$

5. El "Anteproyecto" contiene 17 elementos principales y 176 acciones prioritarias, que se deben implementar cada dos años hasta 2015, o sea de 2008-09, 2010-11, 2012-13 y 2014-15.

6. N. del T. "Efecto de subirse al tren": fenómeno que se produce cuando alguien tiene éxito al aprovechar una oportunidad, haciendo que otros reaccionen, imitándolo y tratando de aprovechar la misma oportunidad. 


\section{China}

Es sorprendente que en 2002 China firmara un acuerdo con la ASEAN sobre planes bien diseñados de asociación económica completa, sólo un año después de haberse afiliado a la OMC en 2001. Esto recibió el nombre de "Acuerdo Marco de Cooperación Económica Completa entre la Asociación de Naciones del Sudeste de Asia y la República Popular China". El "Acuerdo Marco" esbozó los acuerdos a alcanzar en la liberalización del comercio en un periodo de 10 años en lo referente a productos, servicios e inversión, así como los acuerdos sobre ciertos aspectos de la cooperación económica. Además, antes de iniciarse las reducciones del arancel, se acordó poner en práctica un Programa de Cosecha Temprana para acelerar la integración comercial de algunos productos primarios. En el programa se incluyeron productos tales como animales vivos, carne y vísceras comestibles de res, pescado, productos lácteos, otros productos de origen animal, árboles vivos, vegetales comestibles, y frutas y nueces comestibles. Los plazos y la velocidad de reducción y eliminación del arancel dependían de las categorías de productos. Estaba previsto que el programa concluyera en 2006.

El TLC de productos ASEAN-China fue aprobado por ambas partes en 2004 y entró en vigor en julio de 2005. En 2007 se aprobó un TLC ASEAN-China en la esfera de los servicios y entró en vigor en julio de 2007. Un acuerdo de inversión entre la ASEAN y China se firmó en 2009 y entró en vigor en enero de 2010. De esta manera, se logró totalmente el Área de Libre Comercio ASEANChina (ACFTA, por sus siglas en inglés). El ACFTA cubre aproximadamente 1.9 mil millones de personas y US $\$ 6.000$ mil millones en términos de PBI, lo que lo convierte en el área de libre comercio más grande del mundo. Los aranceles deben reducirse a cero para alrededor de 7,000 productos (93\% de los productos comercializables) en 2010 para los países fundadores de la ASEAN. Para los cuatro países que se incorporaron posteriormente a la ASEAN, las aranceles cero se aplicarán en el año 2015.

La influencia del ACFTA es incalculable. China puede disfrutar de productos primarios tropicales, productos minerales, gas natural (de Myanmar) y piezas y componentes de alta tecnología de la ASEAN, mientras la ASEAN puede beneficiarse de productos de consumo baratos y productos de la industria pesada de China. La inversión china en la ASEAN aumentará debido a los lazos comerciales y culturales a través de los chinos en el exterior que viven en la región. Además, el yuan chino penetrará en la ASEAN como moneda comercial. 
Tabla 1

Anteproyecto de comunidad económica de la ASEAN

Cronograma estratégico

\section{Mercado único y base productiva}

\begin{tabular}{|c|c|}
\hline Libre flujo de productos & \\
\hline $\begin{array}{l}\text { Liberalización: } \\
\text { Eliminación de aranceles y de las barreras no } \\
\text { arancelarias }\end{array}$ & $\begin{array}{l}\quad \text { Facilitación: } \\
\text { Integración aduanera; } \\
\text { normas y barreras técnicas para el comercio }\end{array}$ \\
\hline
\end{tabular}

\begin{tabular}{|l|l|}
\hline \multicolumn{1}{|c|}{ Libre flujo de servicios } & \multicolumn{1}{|c|}{ Liberalización: } \\
$\begin{array}{l}\text { Pleno acceso al mercado y tratamiento nacional; } \\
\text { eliminación sustancial de todas las restricciones al } \\
\text { comercio de servicios }\end{array}$
\end{tabular}$\quad$\begin{tabular}{l}
\multicolumn{1}{c|}{\begin{tabular}{c}
\multicolumn{1}{|c|}{ Fación: } \\
ARM sobre los servicios profesionales; \\
intercambio profesional
\end{tabular}} \\
\hline
\end{tabular}

\begin{tabular}{|c|c|}
\hline Libre flujo de la inversión & \\
\hline $\begin{array}{l}\text { Liberalización: } \\
\text { Todas las industrias/ servicios incidentales a esas } \\
\text { industrias para los inversionistas de la ASEAN }\end{array}$ & $\begin{array}{l}\text { Facilitación: } \\
\text { Transparencia; procedimientos dinámicos; } \\
\text { anulación del doble gravamen y promoción } \\
\text { conjunta }\end{array}$ \\
\hline
\end{tabular}

\begin{tabular}{|l|l|}
\hline \multicolumn{1}{|c|}{ Libre flujo de capital } & \multicolumn{1}{c|}{\begin{tabular}{c}
\multicolumn{1}{c|}{ Facilitación: } \\
\hline \multicolumn{1}{|c|}{ Liberalización: } \\
$\begin{array}{l}\text { Relajamiento de las medidas de control del capital en } \\
\text { las inversiones indirectas dentro de la ASEAN }\end{array}$
\end{tabular}} \\
$\begin{array}{l}\text { Intercambio de información; estudios de } \\
\text { impacto e intercambio reteniendo la estructura } \\
\text { de impuestos. }\end{array}$ \\
\hline
\end{tabular}

\begin{tabular}{|c|c|}
\hline Libre flujo de mano de obra calificada & \\
\hline $\begin{array}{l}\text { Liberalización: } \\
\text { Eliminación de la discriminación en el empleo }\end{array}$ & $\begin{array}{l}\text { Facilitación: } \\
\text { Armonización de las normas en la formación y } \\
\text { el entrenamiento; ARM en el adiestramiento } \\
\text { vocacional }\end{array}$ \\
\hline
\end{tabular}

\section{Sectores de integración prioritarios}

El enfoque sectorial para enfocar sus limitados recursos en la integración rápida y profunda en las áreas críticas

Alimentos, agricultura y silvicultura
Ampliar el comercio y la competitividad a largo plazo de los productos
alimenticios, agrícolas y forestales de la ASEAN;
promover la cooperación con organizaciones internacionales,
regionales y el sector privado.

Fuente: Secretaría de la ASEAN.

22 México y la Cuenca del Pacífico. Año 14, núm. 42 / septiembre - diciembre de 2011 


\section{Cronograma estratégico}

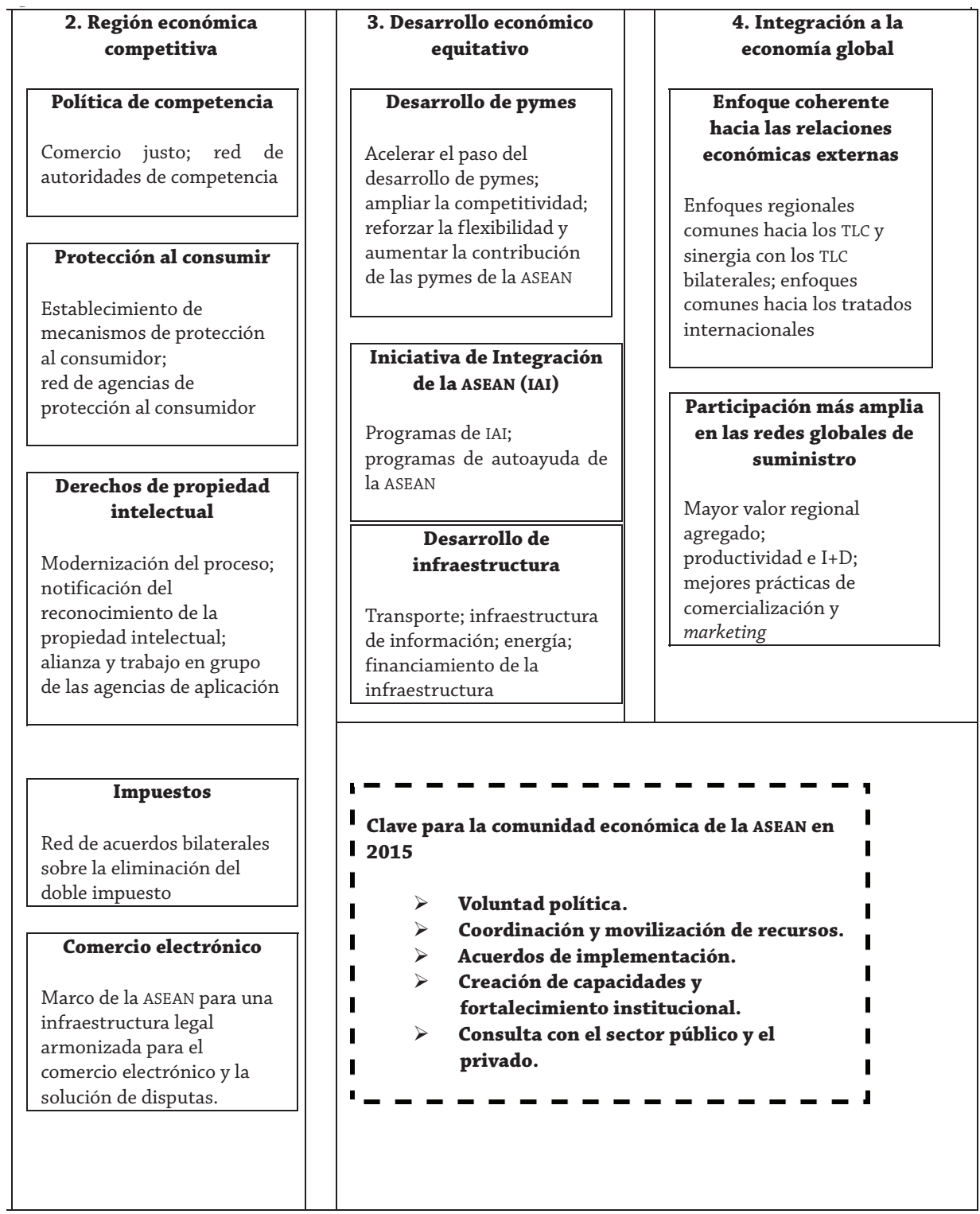


Japón, Corea, Australia y Nueva Zelanda e India

Japón siguió a China en 2003, firmando un acuerdo "Marco para la Asociación Económica Completa entre la Asociación de Naciones del Sudeste de Asia y Japón". También llamado Asociación Económica Completa ASEANJapón (AJCEP, por sus siglas en inglés), este acuerdo es completo e incluye paquetes de liberalización del comercio de productos y servicios, inversión y cooperación económica. Al finalizar el año 2008, todos los participantes habían firmado la AJCEP. Desde agosto de 2010, todos los países miembros de la ASEAN excepto Indonesia la habían ratificado en sus propias legislaturas y la AJCEP entró en vigor.

Japón liberalizó inmediatamente $90 \%$ de los productos comerciales con aranceles cero, y dentro de 10 años lo habrá hecho con 93\%. Entretanto, los seis países de la ASEAN (Brunei, Indonesia, Malasia, Filipinas, Singapur y Tailandia) realizarán eliminaciones inmediatas o reducciones progresivas en $90 \%$ dentro de 10 años. Vietnam promete la eliminación del arancel de hasta 90\% dentro de 15 años, mientras los países $\mathrm{CLM}^{7}$ planean hacerlo con hasta 85\% dentro de 18 años.

Corea siguió a Japón con respecto a las negociaciones con la ASEAN. En el año 2005 ambas partes firmaron un acuerdo marco similar al de China y Japón (“Acuerdo Marco de Cooperación Económica Completa entre los Gobiernos de los Países miembros de la Asociación de Naciones del Sudeste de Asia y la República de Corea"). Con este como base, en 2006 Corea y los países miembros de la ASEAN, excepto Tailandia, firmaron un acuerdo para el comercio de productos. En 2007 se firmó un acuerdo para el comercio de servicios, también con la excepción de Tailandia. Este último país se unió tanto para los productos como para los servicios en febrero de 2009. El acuerdo de inversión entre todos los países de la ASEAN y Corea se firmó en Jeju-Do, Corea, en junio de 2009, cumpliendo así todas las promesas del acuerdo marco. El Área de Libre Comercio ASEAN-Corea (AKFTA, por sus siglas en inglés) para el comercio de productos entró en vigor en julio de 2007, y estipula la eliminación de aranceles de prácticamente todos los artículos para el 2012 entre la ASEAN y Corea. El AKFTA para el comercio de servicios entró en vigor en mayo de 2009.

7. Camboya, RDP Lao y Myanmar. 
Australia y Nueva Zelanda tenían la misma idea en cuanto a un TLC. En 2004 en Vientián, se comprometieron con la "Declaración Conjunta de los Líderes en la Cumbre Conmemorativa ASEAN-Australia y Nueva Zelanda”. En esta declaración, se anunció el inicio de negociaciones sobre un Área de Libre Comercio ASEAN-Australia y Nueva Zelanda (AANZFTA, por sus siglas en inglés). Finalmente, en 2009 se firmó el AANZFTA entre los países mencionados como "Acuerdo de Creación del Área de Libre Comercio ASEAN-Australia Nueva Zelanda" en la Reunión de Ministros de Economía en Cha-am, Tailandia. El AANZFTA es más completo que el AJCEP porque cubre el comercio de productos, servicios (incluyendo financieros y de telecomunicaciones), comercio electrónico, inversión, cooperación económica, y derechos sobre la propiedad intelectual, entre otras áreas. También es un acuerdo de región a región. Este es también el primer TLC negociado de conjunto por Australia y Nueva Zelanda con otra región, la ASEAN en este caso.

El AANZFTA entró en vigor el $1^{\circ}$ de enero de 2010 para ocho países (Brunei, Malasia, Myanmar, Filipinas, Singapur, Vietnam, Australia y Nueva Zelanda). El mismo entró en vigor para Tailandia el 12 de marzo de 2010. Los tres países restantes: Camboya, Indonesia y la RDP Lao, lo ratificarían a finales de ese mismo año.

En lo que respecta a la India, el impulso provino en primer lugar de la Cumbre ASEAN-India en Phnom Penh en 2002, en la que se anunció un "Área de Inversión y Comercio Regional ASEAN-India (RTIA, por sus siglas en inglés)" como un objetivo a largo plazo. Luego, en 2003 siguió el "Acuerdo Marco de Cooperación Económica Completa entre la ASEAN e India”. Este acuerdo marcó el inicio de negociaciones para la creación de un Área de Libre Comercio ASEAN-India (AIFTA, por sus siglas en inglés) en términos de comercio de productos y servicios, inversión y cooperación económica. El acuerdo también incluyó un Programa de Cosecha Temprana de 26 artículos entre 2004 y 2007. Debido a una diferencia de opinión sobre las Reglas de Origen, el programa no se pudo implementar.

La liberalización del comercio de productos se acordó por primera vez entre la ASEAN y la India en 2009 como "Acuerdo para el Comercio de Productos conforme al Acuerdo Marco de Cooperación Económica Completa entre la Asociación de Naciones del Sudeste de Asia y la República de la India”. Otras cuestiones tales como el comercio de servicios y la inversión en el AIFTA se dejaron para negociaciones posteriores, designando el mes de agosto de 2010 como fecha tope para llegar a una conclusión. Actualmente, desde el $1^{\circ}$ de 
enero de 2010, entró en vigor un AIFTA para el comercio de productos con respecto a India, Malasia, Singapur y Tailandia.

\section{Participación de Japón}

Japón asumió enfoques tanto bilaterales como de integración para sus políticas comerciales y de inversión. Para un enfoque bilateral, Japón negocia TLC o Acuerdos de Asociación Económica (EPA, por sus siglas en inglés) con países individuales. El otro enfoque implica que Japón tome la iniciativa de alcanzar la integración económica con diversos países, como la ASEAN+3 (China, Corea y Japón), la ASEAN+6 (Australia, China, India, Corea, Nueva Zelanda y Japón), y el foro para la Cooperación Económica Asia- Pacífico (APEC, por sus siglas en inglés).

\section{EPA bilaterales}

Los acuerdos de Japón son más amplios que los TLC porque cubren no sólo el comercio de productos y servicios, sino también movimientos de mano de obra calificada, contratos con el sector público, propiedad intelectual, medidas proteccionistas,

Los acuerdos de Japón son más amplios que los TLC porque cubren no sólo el comercio de productos y servicios, sino también movimientos de mano de obra calificada, contratos con el sector público, propiedad intelectual, medidas proteccionistas, solución de disputas, inversión y cooperación económica. Es por ello que Japón prefiere el término EPA (Economic Partnership Agreement) al de TLC solución de disputas, inversión y cooperación económica. Es por ello que Japón prefiere el término EPA (Economic Partnership Agreement) al de TLC. En la actualidad Japón tiene firmados 11 EPA, está negociando otros cinco y ha comenzado la investigación de uno más. Los 11 EPA son con Singapur, México, Malasia, Chile, Tailandia, Indonesia, Brunei, la ASEAN, Filipinas, Suiza y Vietnam (tabla 2). Los cinco negociados son con Corea; el Consejo de Cooperación del Golfo (GCC, por sus siglas en inglés) compuesto por el Reino de Bahréin, el Estado de Kuwait, el Sultanato 
de Omán, el Estado de Qatar, el Reino de Arabia Saudita y los Emiratos Árabes Unidos (EAU); India; Australia y Perú. Además, Japón y Mongolia comenzaron la investigación pública y privada conjunta sobre un EPA en junio de 2010 (tabla 3).

Corea es muy activa en la concertación de TLC bilaterales con otros países/ regiones. En estos momentos tiene en vigor cinco TLC, con Chile, Singapur, la ASEAn, India, y la Asociación Europea de Libre Comercio (AELC). Corea concluyó negociaciones de TLC con Estados Unidos y la Unión Europea, y está en negociaciones con ocho países/regiones: Canadá, México, Australia, Nueva Zelanda, Perú, Colombia, Turquía y el GCC. Además, siete países/regiones consideran la formación de TLC: Japón, China, China-Japón, Rusia, Israel, la Unión Aduanera del Sur de África (SACU, por sus siglas en inglés), y Mercosur o el Mercado Común del Sur conformado por Argentina, Brasil, Paraguay y Uruguay.

China también está muy interesada en tener TLC bilaterales. El país firmó e implementó TLC con Chile, Nueva Zelanda, Paquistán, Perú y Singapur así como la ASEAN y ha concluido negociaciones con Costa Rica. China negocia actualmente TLC con Australia, el GCC, Islandia y Noruega.

\section{Esfuerzos de integración}

La crisis financiera asiática de 1997-1998 hizo que fuera necesaria la coordinación y alineación política entre los países asiáticos. La ASEAN invitó a China, Japón y Corea a organizar la primera Cumbre ASEAN+3 en Kuala Lumpur en 1997. Uno de los logros de este esfuerzo fue el esquema financiero Iniciativa de Chiang Mai (CMI, por sus siglas en inglés) creado entre 1999 y 2000. La Cumbre ASEAN+3 en 2004 acordó organizar una Cumbre del Este Asiático (ASEAN+6). La primera Cumbre del Este Asiático (EAS, por sus siglas en inglés) se realizó en Kuala Lumpur en diciembre de 2005. Los 16 participantes en la EAS fueron los 10 países miembros de la ASEAN, más Australia, China, India, Japón, Corea y Nueva Zelanda. La Declaración de la Cumbre de Kuala Lumpur mencionó por primera vez que los países de la EAS ofrecerían una base para "promover la creación de una comunidad en esta región".

Japón tomó la iniciativa bajo el gobierno del Partido Democrático Liberal (tercer gabinete de Koizumi, Abe, Fukuda y Aso entre 2006 y 2009) de ser el arquitecto de una Comunidad del Este Asiático. La iniciativa de esta comunidad propuesta por el gobierno japonés (formulada principalmente por el 


\section{Tabla 2}

Japón: EPA que ya están en vigor

\begin{tabular}{llll}
\hline País o región & Firmado en & En vigor desde & Notas \\
\hline Singapur & Enero de 2002 & 30 de noviembre de 2002 & Modificado en 2007 \\
& & & y 2008 \\
México & Septiembre de 2004 & $1^{\circ}$ de abril de 2005 & $\begin{array}{l}\text { Se agregaron } \\
\text { algunas cuotas de } \\
\end{array}$ \\
& & & aranceles en 2007
\end{tabular}

\begin{tabular}{|c|c|c|c|}
\hline Malasia & Diciembre de 2005 & 13 de julio de 2006 & \\
\hline Chile & Marzo de 2007 & 3 de septiembre de 2007 & \\
\hline Tailandia & Abril de 2007 & $1^{\circ}$ de noviembre de 2007 & \\
\hline Indonesia & Agosto de 2007 & $1^{\circ}$ de julio de 2008 & \\
\hline Brunei & Junio de 2007 & 31 de julio de 2008 & \\
\hline ASEAN & $\begin{array}{l}\text { Todos concluidos en } \\
\text { abril de } 2008\end{array}$ & $\begin{array}{l}\text { Japón, Singapur, RDP Lao, } \\
\text { Vietnam y Myanmar: } 1^{\circ} \text { de } \\
\text { diciembre de 2008; } \\
\text { Brunei: } 1^{\circ} \text { de enero de } 2009 ; \\
\text { Malasia: } 1^{\circ} \text { de febrero de } 2009 ; \\
\text { Tailandia: } 1^{\circ} \text { de junio de } 2009 ; \\
\text { Camboya: } 1^{\circ} \text { de diciembre de } \\
2009 \text {; yilipinas: } 1^{\circ} \text { de julio de } \\
2010 \text {. }\end{array}$ & $\begin{array}{l}\text { Indonesia, aún sin } \\
\text { ratificar }\end{array}$ \\
\hline Filipinas & Septiembre de 2006 & 11 de diciembre de 2008 & \\
\hline Suiza & Febrero de 2009 & $1^{\circ}$ de septiembre de 2009 & \\
\hline Vietnam & Diciembre de 2008 & $1^{\circ}$ de octubre de 2009 & \\
\hline
\end{tabular}

Fuente: Ministerio de Asuntos Exteriores.

Ministerio de Economía, Comercio e Industria) consiste en dos partes: liberalización e integración a través de una Asociación Económica Completa para el Este Asiático (CEPEA, por sus siglas en inglés) e investigación de política y creación de capacidades a través de un Instituto de Investigación Económica de la ASEAN y el Este Asiático (ERIA, por sus siglas en inglés). La iniciativa fue propuesta a la EAS en enero de 2007 (Kagami, 2008). La creación del ERIA, una nueva organización de investigación internacional, se aprobó en la EAS de Singapur en noviembre de 2007 y el instituto se creó en Yakarta en junio de 2008 con el apoyo financiero del gobierno de Japón. Posteriormente, el 
Tabla 3

Japón: EPA en negociación

\begin{tabular}{|c|c|c|}
\hline País o región & $\begin{array}{l}\text { Las negociaciones } \\
\text { comenzaron en }\end{array}$ & Notas \\
\hline Corea & Diciembre de 2003 & $\begin{array}{l}\text { Las negociaciones se suspendieron en } \\
\text { noviembre de } 2004 \text { hasta el presente. }\end{array}$ \\
\hline $\begin{array}{l}\text { Consejo de } \\
\text { Cooperación del } \\
\text { Golfo (GCC) }\end{array}$ & Septiembre de 2006 & $\begin{array}{l}\text { El GCC compuesto por Bahréin, Kuwait, } \\
\text { Omán, Qatar, Arabia Saudita y Emiratos } \\
\text { Árabes Unidos. } \\
\text { Este es un Tratado de Libre Comercio (TLC). }\end{array}$ \\
\hline India & Enero de 2007 & \\
\hline Australia & Abril de 2007 & \\
\hline Perú & Mayo de 2009 & \\
\hline Mongolia & Junio de $2010^{*}$ & $\begin{array}{l}\text { * Se inició la investigación conjunta } \\
\text { pública-privada sobre la JMEPA. }\end{array}$ \\
\hline
\end{tabular}

Fuente: Ministerio de Asuntos Exteriores.

gobierno de Indonesia respaldó el ERIA como una organización internacional en diciembre de 2008.

El ERIA tiene dos objetivos: primero la investigación política y la recomendación de los resultados de la investigación a reuniones ministeriales o cumbres (EAS), y segundo la puesta en marcha de programas de creación de capacidades para los encargados de elaborar las políticas, sobre todo en los últimos países incorporados a la ASEAN (CLMV). ${ }^{8}$ Las actividades de investigación del ERIA en 2009 cubrieron 10 proyectos que están profundamente relacionados con tres cuestiones fundamentales: integración, diferencias de desarrollo y desarrollo sostenible. Uno de los proyectos más llamativos es el Plan Integral de Desarrollo Asiático (CADP, por sus siglas en inglés) en cooperación con el Banco de Desarrollo Asiático ( $\mathrm{ADB}$, por sus siglas en inglés) y la Secretaría de la ASEAN.

Este último tiene como objetivo un plan económico completo del este asiático que aceleraría el desarrollo de la región y la expansión del mercado, como un motor de crecimiento para reforzar la economía mundial que ha sido severamente dañada por el shock Lehman y la crisis financiera en Grecia. El CADP proporciona un magnífico diseño para la infraestructura económica y la

8. Camboya, RDP Lao, Myanmar y Vietnam. 
distribución espacial industrial, y también incluye un análisis de simulación modelada sobre los efectos económicos de las construcciones planificadas de grandes corredores de red.

Los programas de creación de capacidades consisten en enviar a conferencistas a los países CLMV y en cursos de formación, tales como el curso de seis meses para burócratas de CLMV impartido por la IDE Advanced School (IDEAS, por sus siglas en inglés) en Japón. Utilizando estos instrumentos, Japón apoya fuertemente dicha integración económica como Comunidad Económica de la ASEAN para el año 2015 y la Comunidad del Este Asiático (ASEAN+6) en el futuro.

Durante el actual gobierno del Partido Demócrata, la situación ha cambiado considerablemente debido a: 1 . El incidente de la colisión de barcos en Senkaku ocurrida en octubre de 2010, 2. Japón fue sede de la Cumbre de la APEC (Foro de Cooperación Económica de Asia-Pacífico) en noviembre de 2010 en Yokohama, y 3. El rápido movimiento de Corea para la firma de TLC, sobre todo con Estados Unidos y la Unión Europea. Un barco pesquero chino chocó intencionalmente con un patrullero de guardacostas de Japón cerca de las Islas Senkaku sobre las que ambos países reclaman soberanía territorial. El capitán chino fue detenido, pero más tarde liberado. Esto dio lugar a una acalorada discusión en torno al problema territorial pero el gobierno de Japón lo negó, ya que las islas son japonesas.

En la Cumbre de la APEC, sus 21 economías debatieron sobre el Área de Libre Comercio de Asia-Pacífico (FTAAP, por sus siglas en inglés). Además, Corea firmó un TLC bilateral con Estados Unidos el 30 de junio de 2007, y con la Unión Europea el 6 de octubre de 2010. Esto generó un sentimiento de impaciencia de que se estaba dejando a un lado a Japón, sobre todo en el grupo comercial. Estos factores afectaron la política comercial de Japón, llevando a la promoción de un nuevo enfoque.

De repente, el gobierno japonés comenzó a hablar de su participación en el Acuerdo de la Asociación Transpacífica (TPP, por sus siglas en inglés). El TPP entró en vigor en 2006 con Brunei, Chile, Nueva Zelanda y Singapur como sus miembros originales. Estados Unidos (al igual que Australia, Malasia, Perú y Vietnam) mostró interés en unirse. Si Japón se une, puede formar un vínculo estrecho con Estados Unidos aun cuando Japón enfrenta grandes problemas en la agricultura. Sin embargo, el propio TPP tiene méritos porque proporciona una buena plataforma para una mayor integración económica, que cubre países transpacíficos. En cierto modo, es un primer paso que conduce al FTAAP. 


\section{Comentarios a modo de conclusión}

El este asiático se ha convertido en el mayor centro de producción y mercado consumidor del mundo. La ASEAN pronto se transformará en una comunidad económica llamada AEC. Además, la ASEAN se ha ido convirtiendo en un centro de comercio e inversión en el este asiático, debido a que Australia, China, India, Japón, Corea y Nueva Zelanda han firmado TLC/EPA con la región. La propia ASEAN firmó TLC entre sus miembros en forma de AFTA. Esto significa que la región se está convirtiendo en el área comercial más grande y libre del mundo. Por consiguiente, el comercio y la inversión intrarregional aumentarán considerablemente (figura A).

Sin embargo también surgen algunos problemas, entre ellos los siguientes:

1. Efecto del plato de espagueti. En lo referente a los 10 países miembros de la ASEAN, tienen AFTA entre ellos y ahora disfrutan de TLC/EPA con China, Japón, Corea, Australia y Nueva Zelanda, e India. Además, estos países vecinos tienen TLC/EPA bilaterales con algunos países de la ASEAN. Por ejemplo, Japón tiene EPA bilaterales con Brunei, Indonesia, Malasia, Filipinas, Singapur, Tailandia y Vietnam (véase la figura A). Corea mantiene TLC bilateral con Singapur; China e India también con Singapur; Australia con Singapur y Tailandia; y Nueva Zelanda con Malasia, Singapur y Tailandia. Además, seis países también se encuentran preparando TLC/EPA entre sí. Por ejemplo, Japón negocia EPA bilaterales con Corea, India y Australia, mientras China completó su TLC con Nueva Zelanda y negocia un TLC con Australia. Existe una enorme confusión sobre cuál TLC/EPA se aplicará. Incluso los agentes de aduana no saben qué arancel deben aplicar a un producto específico y qué normas de origen son relevantes, debido a que se reproducen tantos acuerdos sin elaborarse de manera integrada. A esto se le llama "efecto de plato de espagueti".

2. Megacompetencia industrial. La ASEAN se ha convertido en un gran centro de comercio e inversión. Con respecto a las industrias manufactureras, esto representa una atractiva oportunidad para que las naciones vecinas inviertan en uno de los países de la ASEAN con el objetivo de fabricar productos y luego exportarlos a otros países del área. Por ejemplo, una compañía de electrónica china puede invertir en Tailandia para hacer productos finales. Teóricamente, esta fábrica chino-tailandesa puede elaborar estos productos importando piezas y componentes de China con aranceles cero usando el ACFTA, e importar también otras piezas y componentes de países de la ASEAN sin aranceles usando el AFTA. Luego la fábrica puede exportar los productos 
finales, por ejemplo a la India, sin pagar los aranceles de aquel país usando el AIFTA. Australia, India, Japón, Corea y Nueva Zelanda pueden operar de la misma manera utilizando la ASEAN como base de producción. Esto significa que las afluencias de la inversión extranjera directa (FDI, por sus siglas en inglés) hacia la ASEAN aumentarán considerablemente desde estos países vecinos.

Sin embargo, esto significa que la competencia entre ellos será mucho más severa. En particular, los países de la ASEAN tienen que competir con estas naciones vecinas. Tienen que buscar sus propios sectores industriales convenientes o algunos procesos de producción que ofrezcan ventajas comparativas. El resultado será el reemplazo, reasignación, reubicación, realineamiento geográfico y reorganización de la industria. En otras palabras, en toda la región tiene lugar la división vertical y horizontal del trabajo. Ha llegado la era de la megacompetencia. En este contexto, se debe prestar especial atención a los últimos países que se incorporaron a la ASEAN, o sea a los países CLMV (Ishida, 2010; Kagami, 2010; y Kawada, 2011).

Figura A

La ASEAN como centro del comercio

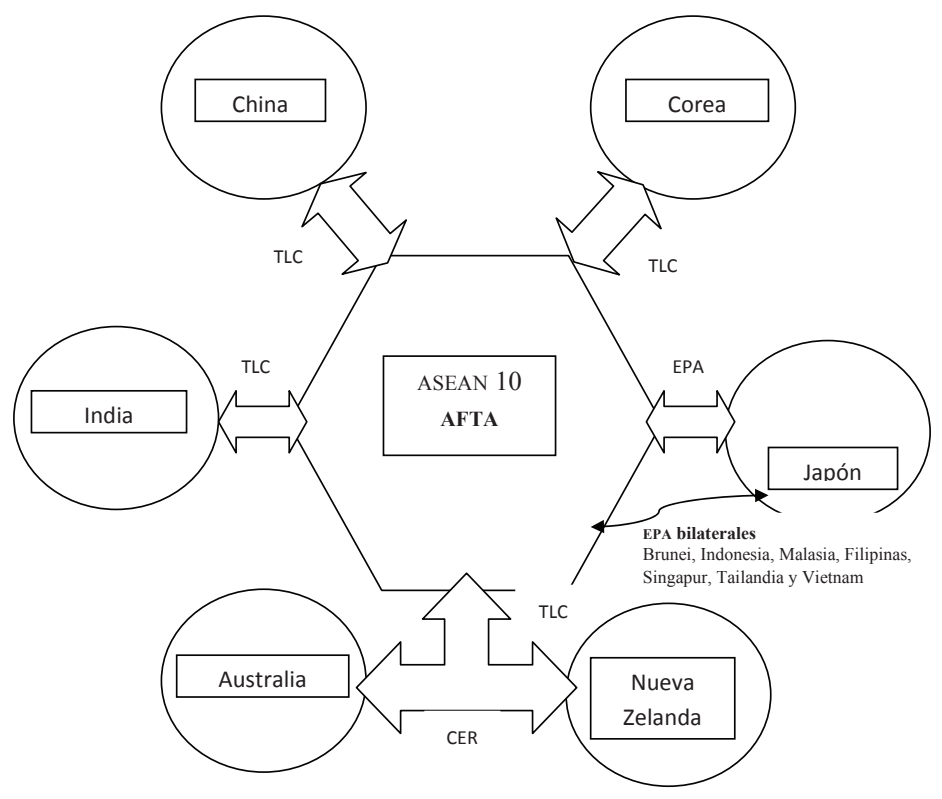

Fuente: dibujo del autor. 
3. Agricultura. Lo mismo ocurrirá en la agricultura. Se dice que los países agrícolas pequeños, pero económicos, pueden salir ganando en un mundo totalmente liberalizado mediante la exportación de sus productos agrícolas. Sin embargo, esto no es necesariamente así, debido a que en ocasiones esos países no son capaces de mantener un suministro estable y en volúmenes grandes con pronta entrega. En cambio, los productos agrícolas de Nueva Zelanda o Australia pueden dominar en la región. No obstante, por supuesto, que los TLC/EPA pueden permitir la inclusión de excepciones en la eliminación de aranceles para categorías sensibles, tales como los productos agrícolas. De todos modos, la reasignación de la agricultura es inevitable y aparecerán nuevos tipos de agricultura, tales como el cultivo contratado transfronterizo.

4. Preparación de infraestructura. El desarrollo económico requiere modificaciones en la infraestructura, no sólo en la dura sino también en la suave. Las construcciones de infraestructura dura incluyen caminos y autopistas, puertos y aeropuertos, electricidad y abastecimiento de agua, e instalaciones de telecomunicaciones. La infraestructura suave se refiere a arreglos institucionales tales como leyes y los sistemas de ejecución de la ley, integración aduanera, estandarización de normas técnicas y la educación y capacitación de los recursos humanos. Estos están ausentes, especialmente el último rincón de los países de la ASEAN. El Banco de Desarrollo Asiático (ADB) ha puesto en marcha algunos planes de construcción de infraestructura para la Subregión del Gran Mekong (GMS) que incluye los países CLMV (Camboya, RDP Lao, Myanmar y Vietnam), Tailandia, la provincia de Yunnuan y la región autónoma de China Guangxi Zhuang.

En lo concerniente a la construcción de una red carretera entre estos países y regiones, existen planes bien conocidos de corredores, tales como el Corredor Norte-Sur (Kumning, China-Bangkok, Tailandia pasando por RDP Lao), Corredor Este-Oeste (Da Nang, Vietnam-Mawlamyine, Myanmar pasando por RDP Lao y Tailandia), y el Corredor Sur (ciudad de Ho Chi Minh, Vietnam-Dawei, Myanmar pasando por Camboya y Tailandia). Algunos de los proyectos recomendados por el ABD han sido parcialmente terminados pero algunos no han sido iniciados aún (véase Menon y Melendez, 2011). El desarrollo de GMS es un elemento clave para el fortalecimiento no sólo de los vínculos económicos de la ASEAN, sino también para la integración del este de Asia.

5. Cuestiones relacionadas con la fuerza de trabajo y la migración. Si existe una diferencia en cuanto a los ingresos, las personas emigrarán ilegalmente 
para buscar mayores ingresos. Se dice, por ejemplo, que Tailandia tiene más de dos millones de trabajadores que emigraron ilegalmente de países vecinos. La liberalización puede hacer que se amplíe la diferencia de ingresos entre países. Los programas, como la Iniciativa para la Integración de la ASEAN en el "Anteproyecto" de la AEC son necesarios para reducir la diferencia de ingresos entre los países fundadores y los países incorporados posteriormente a la ASEAN. Al mismo tiempo, los programas para el readiestramiento y la reeducación de trabajadores no calificados son una necesidad fundamental.

6. Disputas fronterizas y políticas. Hay varias preocupaciones por cuestiones políticas en cuanto al logro de la integración sin problemas. El gobierno militar de Myanmar realizó unas elecciones generales el 7 de noviembre de 2010, pero existe gran preocupación de que las mismas no se realizaron democráticamente. También se has suscitado disputas fronterizas, como las que tienen lugar entre Tailandia y Camboya por el Templo Preah Vihear, entre China y Vietnam por las Islas Paracel, y también entre países vecinos por las Islas Spratly. Aun así, hay esperanza de que estas cuestiones no dificulten el proceso de integración, como la AEC.

7. Destrucción ambiental. Como se ha visto en las experiencias de países industrializados avanzados, la destrucción ambiental es inevitable cuando se acelera el desarrollo. Japón, por ejemplo, era celebre por la llamada "enfermedad de Minamata" causada por la contaminación del agua en los años 1950 y 1960. La misma se debió a que una compañía química vertió mercurio en la bahía Minamata. Si se acelera el crecimiento económico, también aumentarán la polución del aire y el agua, la contaminación de la tierra y la erosión de suelo en la región. Se necesitan "economías verdes" para ayudar a liberar el ambiente de la destrucción. El este asiático está orgulloso de su belleza natural y de sus ricos recursos turísticos, incluyendo el hecho de que alberga el décimo río más largo del mundo, el Mekong. Deberíamos proteger nuestros recursos naturales y promover el uso adecuado de este canal.

8. Participación de Estados Unidos y Rusia en la EAs. La Reunión de Ministros de Asuntos Exteriores de la ASEAN celebrada en Hanoi el 20 de julio de 2010, acordó que la ASEAN invitara a Estados Unidos y Rusia a hacerse miembros de la Cumbre del Este Asiático (Nueva EAS o ASEAN+8), posiblemente en el año 2011. La razón principal de esta decisión podría radicar en cuestiones de seguridad. En particular, la ASEAN está preocupada por la expansión de la marina china tanto en el mar del este como del sur de China y el océano Indico. Se dice que Rusia desea ser miembro de la EAS desde su creación. 
La participación de las dos grandes potencias cambiaría completamente el equilibrio en la región.

Resulta muy natural que la región asiática quiera formar su propio TLC, a la luz de ejemplos como el Tratado de Libre Comercio de América del Norte (NAFTA, por sus siglas en inglés) en la región norteamericana y Mercosur en Sudamérica. Parece que China promueve la idea de la ASEAN+3 y, como se explicó anteriormente en este trabajo, Japón quiere la ASEAN+6. (El gabinete Hatoyama mencionó una "Comunidad del Este Asiático" pero probablemente se refería a la ASEAN+3.) Al menos, la participación de dos grandes potencias afectará las iniciativas japonesas y chinas de formar una comunidad integrada dentro del este asiático.

China parece preferir el esquema ASEAN +3 al ASEAN +6 , esto debido a que no tiene buenas relaciones diplomáticas con la India a causa a su frontera común y a las armas nucleares, y con Japón debido a sus razones históricas. Por otra parte, otro aspecto son las recientes relaciones entre China y Estados Unidos. Los dos países han desarrollado una confianza mutua a través de las conversaciones "Six-Party" (China, Japón, Corea del Norte, Rusia, Corea del Sur y Estados Unidos) sobre el problema nuclear de Corea de Norte en el periodo 2003-2007. China ha presidido todas estas seis pláticas experimentando las habilidades de negociación y actitudes sensibles hacia Estados Unidos. Lo anterior significa que, salvo por los asuntos militares y seguridad, Estados Unidos parece asumir la misma posición que China con bastante facilidad. Si se uniera a la EAS, tal situación podría ser una amenaza, especialmente para la India y Japón.

10. El movimiento TPP. El actual gabinete de Kan parece contemplar el TPP y el FTAAP. Existen opiniones positivas para el TPP entre los círculos empresariales y economistas en Japón (Hatakeyama, 2011). La unión de Japón al TPP puede enviar una mala señal a los países de Asia del este, como que Japón deja Asia y va hacia el Pacífico. Esto animaría a China porque así podría ampliar su influencia económica en la región, sin Japón. Por eso, este autor piensa que deberíamos considerar seriamente la formación de la Comunidad Económica de la ASEAN (AEC) primero y la integración de la ASEAN+6 después. Pienso así porque en estos momentos el área es un centro de producción y uno de los mercados de demanda más grandes del mundo, recordemos que en 2008 la participación de la región ASEAN+6 en las exportaciones mundiales de productos finales fue $33 \%$ y la de sus importaciones de $19 \%$. miv 


\section{Referencias}

Hatakeyama, Noboru, "Breaking up the Japanese Economic Stoppage by TPP (en japonés)”, Chuokoron, mayo de 2011, Chuokoron-sha, 2011.

Ishida, Masami (ed.), Outlook of the Border Economies in the Mekong Region (en japonés), Institute of Developing Economies (Japan External Trade Organization), 2010.

Kagami, Mitsuhiro, "Policy Recommendations of the ERIA Study Project (FY2007)", BRC Discussion Paper Series núm. 1, Bangkok Research Center, IDE-JETRO, 2008.

Kagami, Mitsuhiro (ed.), Economic Relations of China, Japan and Korea with the Mekong River Basin Countries, BRC Research Report núm. 3, Bangkok Research Center, IDE-JETRO, 2010. Disponible en: http://www.ide.go.jp/ English/Publish/Download/Brc/index.html.

Kawada, Atsusuke, Greater Mekong Subregion: Integrated Development by Fixing Infrastructure (en japonés), Keiso Shobo, 2011.

Menon, Jason y Anna Cassandra Melendez, "Trade and Investment in the Greater Mekong Subregion: Remaining Challenges and the Unfinished Policy Agenda", ADB Working Paper Series on Regional Economic Integration núm. 78, mayo de 2011, Asian Development Bank, 2011.

Páginas Web

http://www.aseansec.org/4920htm.

http://www.mofa.go.jp/policy/economy/fta/index.html.

http://www.mofat.go.kr/english/econtrade/fta/issues/index2.jsp.

http://english.mofcom.gov.cn/aarticle/newsrelease/significant-

news/201003/20100306812606.html.

http://www.commerce.nic.in/trade/international_ta.asp?id=2\&trade=i. http://www.dfat.gov.au/trade/ftas.html.

http://www.mfat.govt.nz/Trade-and-Economic-Relations/Trade-Agree-

ments/index.php.

http://www.adb.org/economics/.

http://www.eria.org/publications/policy.html. 